\title{
AUTOREN Autonomes Fahren auf dem Busbetriebshof
}

Andreas Lauber, M. Sc. ist Wissenschaftlicher Mitarbeiter am Institut für Technik der Informationsverarbeitung (ITIV) des Karlsruher Institut für Technologie (KIT).

Prof. Dr.-Ing. Eric Sax ist Institutsleiter des Instituts für Technik der Informationsverarbeitung (ITIV) des Karlsruher Institut für Technologie (KIT).
Markus Wiedemann ist Leiter der Kraftfahrzeugwerkstätten bei den Stuttgarter Straßenbahnen $A G$ (SSB).
Flächendeckendes autonomes oder auch hochautomatisiertes Fahren ist derzeit aufgrund verschiedener rechtlicher Hürden im Personenverkehr noch nicht umsetzbar. Im Bereich von nichtöffentlichem Verkehr sind aber heute schon verschiedene Anwendungsfelder denkbar. Bei Logistikbetrieben, ÖPNV-Betriebshöfen und Containerterminals bietet sich durch die Teilautomatisierung von Betriebsabläufen die Chance, Kosten zu optimieren. Eine Studie des KIT bei den SSB zeigt, wie autonomes Fahren auf Busbetriebshöfen funktionieren und zur Kostensenkung beitragen kann.




1 MOTIVATION

2 RECHTLICHE ASPEKTE

3 BETRIEBSABLÄUFE

4 REALISIERUNG DES AUTONOMEN FAHRENS

5 WIRTSCHAFTLICHE INTERESSEN BEI DER AUTOMATION VON

BETRIEBSABLÄUFEN

6 ZUSAMMENFASSUNG

\section{MOTIVATION}

Im öffentlichen Personennahverkehr werden die Fahrzeuge nach der Rückkehr aus dem Betrieb für die nächste Schicht in den Depots vorbereitet. Speziell bei Bussen umfassen die Aktivitäten im Depot kleinere Wartungs- und Inspektionstätigkeiten, insbesondere Betankung, Waschen und Innenreinigung der Fahrzeuge. Für die genannten Tätigkeiten werden die Fahrzeuge nach jedem Arbeitsschritt von einem Mitarbeiter zu den verschiedenen Stationen gefahren, an denen die einzelnen Dienste durchgeführt werden. So sind neben den Mitarbeitern an den Stationen ebenfalls Mitarbeiter erforderlich, die die Fahrzeuge auf dem Betriebshof bewegen. Auf einem untersuchten Busbetriebshof, dem SSBDepot in Stuttgart-Gaisburg, bindet der Einsatz von 150 Bussen jeden Tag Personal, das durch automatisiertes Fahren entlastet werden kann, wodurch sich der Personalaufwand reduziert. Durch den Einsatz von Standardtechnologien soll erreicht werden, dass die Busse sowohl auf dem Betriebshof als auch später auf der öffentlichen Straße autonom fahren können.

\section{RECHTLICHE ASPEKTE}

Das automatisierte Fahren auf Betriebsgeländen macht dabei im Gegensatz zum Einsatz im öffentlichen Verkehrsbereich bereits heute Sinn, da davon ausgegangen werden kann, dass es sich um einen abgeschlossenen und eingezäunten Bereich handelt. Obwohl sich die Fahrzeuge an die geltenden Verkehrsregeln der Straßenverkehrsordnung halten (beispielsweise rechts vor links, Ampeln, Geschwindigkeitsbegrenzung), entfallen viele nicht vorgesehene Situationen durch Passanten oder andere Verkehrsteilnehmer. Spielende Kinder, die einem Ball nachlaufen, kommen auf dem Betriebshof nicht vor. Zur Demonstration des autonomen Fahrens wurde der Betriebshof in Stuttgart-Gaisburg untersucht. Dieser Betriebshof der SSB versorgt die Stuttgarter Innenstadt mit Linienbussen.

\section{BETRIEBSABLÄUFE}

Bei den Linienbussen der SSB umfassen die Aktivitäten im Betriebshof eine Testfahrt, kleinere Wartungs- und Inspektionsarbeiten, Betankung, Waschen und eine grobe Innenreinigung der Fahrzeuge. Diese Arbeitsschritte werden an verschiedenen Stationen von qualifizierten Facharbeitern durchgeführt. Zusätzlich sind diese Facharbeiter durch die Fahrtätigkeiten gebunden, bei denen der Bus zu den Stationen bewegt wird. Der Einsatz von 150 Bussen pro Tag bindet große Personalressourcen. Ziel ist es, die verfügbare Arbeitszeit des Personals durch autonome Fahrten für andere Tätigkeiten frei zu bekommen.

Die auf dem Betriebshof Stuttgart-Gaisburg identifizierten Betriebsabläufe der SSB sind in die Karte des Betriebshofs in BILD 1 eingezeichnet und können in neun Arbeitsschritte zusammengefasst werden. Die identifizierten Betriebsabläufe sind in TABELLE 1 mit ihren evaluierten durchschnittlichen Durchführungszeiten aufgelistet und entsprechen der Nummerierung aus BILD 1. Zum Ende ihres Dienstes fahren die Busfahrer der SSB ihre Dienstfahrzeuge auf den Betriebshof und stellen diese auf speziellen Abstellspuren (1) ab. Die abgestellten Busse werden anschließend vom Wartungspersonal entgegengenommen und zur Wartungshalle gefahren (2). Diese kurze Fahrtstrecke dient der SSB unter anderem als

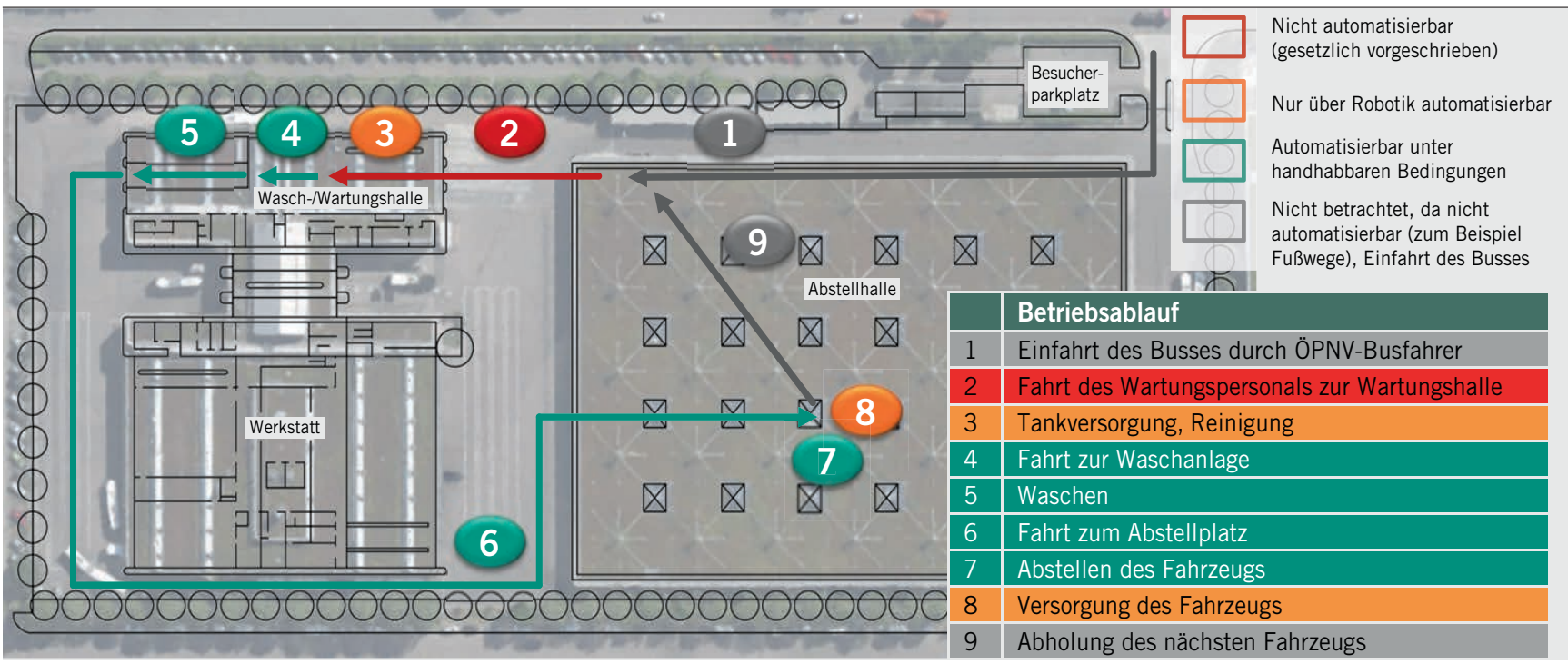

BILD 1 Draufsicht des Betriebshofs Stuttgart-Gaisburg mit Markierung der automatisierbaren Betriebsabläufe (@ ITIV) 


\begin{tabular}{|c|c|c|}
\hline & Betriebsablauf & $\begin{array}{l}\text { Durchschnittliche } \\
\text { Zeit [min] }\end{array}$ \\
\hline 1 & Einfahrt des Busses durch SSB-Busfahrer & \\
\hline 2 & Fahrt des Wartungspersonals zur Wartungshalle & 1 \\
\hline 3 & Tankversorgung, Reinigung & 7 \\
\hline 4 & Fahrt zur Waschanlage & 0,5 \\
\hline 5 & Außenreinigung & 4 \\
\hline 6 & Fahrt zum Abstellplatz & 3 \\
\hline 7 & Abstellen des Fahrzeugs & 0,25 \\
\hline 8 & Versorgung des Fahrzeugs & 0,25 \\
\hline 9 & Abholung des nächsten Fahrzeugs (Fußweg) & 2 \\
\hline
\end{tabular}

TABELLE 1 Übersicht der Betriebsabläufe mit Zeitdauer am Beispiel StuttgartGaisburg (@ ITIV)

implizite Testfahrt zur Sicherung der Halterverantwortung nach StVZO [1]. Innerhalb der Wartungshalle (3) werden neben kleineren Instandhaltungs- und Inspektionstätigkeiten die Fahrzeuge zum einen mit Flüssigkeiten (Diesel, Öl, Ad-Blue etc.) versorgt. Zum anderen wird der Innenraum der Busse gereinigt und auf Mängel untersucht, welche eine Gültigkeit der Betriebserlaubnis gefährden (fehlender Nothammer, Kinnschutz etc.). Im nächsten Schritt werden sie zur Waschanlage gefahren (4) und dort von außen gereinigt (5). Danach werden die Busse um die Werkstattgebäude gefahren (6) und anschließend auf einem zugewiesenen Standplatz abgestellt (7). Hier werden das Abblendlicht und die Zündung abgeschaltet und die Türen zum Schutz vor Tieren geschlossen. Abschließend versorgt der Wartungsmitarbeiter die Fahrzeuge zur Vorkonditionierung mit externer Druckluft (8). Dieser Schritt dient zur schnelleren Einsatzbereitschaft der Fahrzeuge am nächsten Morgen und zur Lärmreduktion, da für einen ausreichenden Betriebsdruck der Bremsanlage ein laufender Motor vermieden wird. In Schritt (9) wird der nächste Bus abgeholt und die Betriebsabläufe beginnen von vorne.

Es wird neben der Tankversorgung und Innenreinigung (3) besonders in den folgenden drei Bereichen unproduktiv gearbeitet:

- Außenreinigung (5) in der Waschanlage

- Fahrt zum Abstellplatz (6)

- Fußweg zum Abholen des nächsten Busses (9).

In diesen Arbeitsschritten steckt somit ein hohes Optimierungspotenzial durch das autonome Fahren und den damit verbundenen Wegfall von Fußwegen. Die Fahrt zur Waschanlage, die Außenreinigung, die Fahrt zum Abstellplatz ebenso wie der Abstellvorgang sind durch entsprechend ausgestattete Fahrzeuge vollständig automatisierbar. Das Betanken, die Innenreinigung und Versorgung der Busse mit Druckluft können in zukünftigen Szenarien Industrieroboter übernehmen. Von der Automatisierung ausgeschlossen bleibt lediglich die Einfahrt in die Wartungshalle (2), da sie implizit als vom Gesetzgeber vorgeschriebene Testfahrt gilt. Auf dieser Strecke werden Bremsen und Lenkung getestet, um festzustellen, dass das Fahrzeug in einem verkehrssicheren Zustand ist.

\section{REALISIERUNG DES AUTONOMEN FAHRENS}

Damit die Funktionalität des automatisierten Fahrens von den Fortschritten der Entwicklung im allgemeinen Nutzfahrzeugbereich partizipieren kann und nicht auf den Betriebshof beschränkt ist, baut das System auf Standardtechnologien für automatisierte Fahrzeuge auf und ist kompatibel mit der normalen Straßenverkehrsinfrastruktur außerhalb des Betriebsgeländes. Die besonderen Herausforderungen beim Beschleunigen, Bremsen und Lenken können über die Ausstattung der Busse mit GPS-, Kamera-, Radarund Ultraschallsystemen gelöst werden. Eine solche Sensorik ist bereits serienmäßig in den Fahrzeugen für die Ortung, Spurhaltung und Abstandswarnung verbaut. Die Verarbeitung dieser Informationen in der sogenannten Sensorfusion erlaubt eine genaue Spurführung und die Erkennung von Hindernissen, wie dies zum Beispiel im Demonstrator-Fahrzeug Future Bus von Daimler [2] bereits gezeigt wurde.

Der Einsatz von Standardtechnologien ermöglicht es außerdem, dass es ausreichend ist, wenn Verkehrsschilder, Ampeln und Markierungen auf dem Betriebshof der herkömmlichen Verkehrsinfrastruktur entsprechen. Um eine Längs- und Querregelung zu erlauben, müssen somit die Abstellflächen und Fahrwege lediglich wie im öffentlichen Straßenverkehr gekennzeichnet sein [3]. Zudem sind Standardtechnologien besonders im öffentlichen Personennahverkehr wertvoll, da Neuanschaffungen europaweit ausgeschrieben werden müssen und damit herstellerspezifische Lösungen nicht für alle eingesetzten Busse (verschiedener Hersteller) identisch sind. Die Busse müssen herstellerunabhängig auf dem Betriebshof automatisiert fahren können, ohne eine proprietäre Lösung nachrüsten zu müssen.

Im Unterschied zum Straßenverkehr müssen auf dem Betriebshof jedoch zwei Arbeitsschritte gesondert betrachtet werden. Zum einen die Einfahrt in die mehrspurige Abstellhalle, zum anderen die Außenreinigung. Bei der Auswahl der Fahrspur in der Abstellfläche, BILD 1 (7), ermittelt der Bus über die Ampelkennzeichnung der Fahrspur die korrekte Fahrspur. Wie an einer Mautstelle wird durch diese Fahrstreifensignalisierung eine Spur freigegeben (grüne Ampel), BILD 2. In gesperrte (rote) Spuren darf der Bus nicht einfahren. BILD 2 zeigt des Weiteren, dass ein Überblicken aller Fahrstreifen aufgrund des Blickwinkels und der Anzahl der Fahrspuren schwierig ist. Dies ist jedoch Voraussetzung für die Erkennung des jeweils freigegebenen Fahrstreifens. Alternativ muss der Bus an der Abstellfläche vorbeifahren und beim ersten grünen Lichtzeichen in die Fahrspur abbiegen. Dadurch kann das Problem der Lichtzeichenerkennung und des großen Blickwinkels gelöst werden. Ein solcher Blickwinkel bei der Einfahrt ist zwar

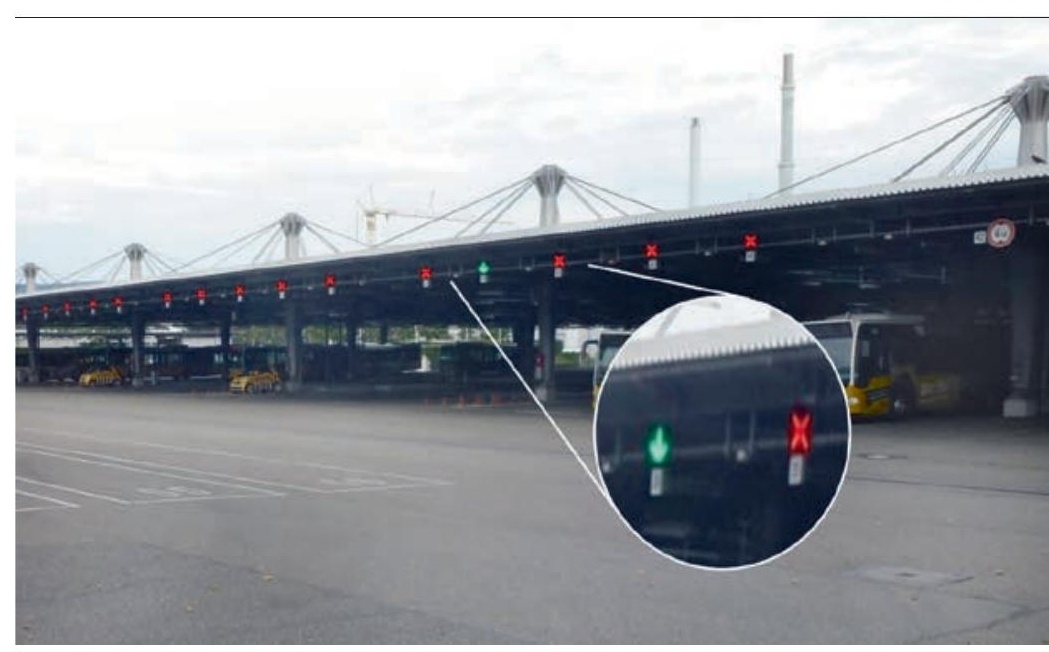

BILD 2 Kennzeichnung der Abstellspuren durch Ampeln (@ ITIV) 
bei Mautstellen unüblich, aber dennoch nicht ausgeschlossen und muss daher auch von autonomen Fahrzeugen im Straßenverkehr beherrscht werden.

Im Gegensatz dazu ist die Außenreinigung, BILD 1 (5), für das automatisierte Fahren auf dem Betriebshof als abweichende Funktion zur Straßenfunktionalität zu sehen. Das Fahren in Waschanlagen mit Schaum und Waschbürsten ist im Normalbetrieb eines autonomen Fahrzeugs nicht vorgesehen. Besonders schwierig ist hierbei zum einen der Umgang mit Schaum auf den Sensoren, die diese verdecken, zum anderen werden Waschbürsten, die sich nahe am Fahrzeug bewegen, als Hindernis erkannt. Um dies zu vermeiden, wird auf eine in Fahrtrichtung bewegbare Portalwaschanlage umgestellt. Diese reinigt dann das sicher stehende Fahrzeug.

Ein wichtiger Punkt für den Einsatz auf Betriebshöfen des öffentlichen Nahverkehrs, aber auch bei Speditionen, ist der zuverlässige Einsatz rund um die Uhr. Es muss vermieden werden, dass durch einen Ausfall des automatisierten Betriebshofs das Ausrücken der Fahrzeuge zu Dienstbeginn nicht möglich ist. Für eine hohe Zuverlässigkeit und Verfügbarkeit bei schlechten Wetterbedingungen (Regen, Schnee, Nebel) werden daher auf dem Betriebshof redundante Maßnahmen vorgenommen. Beispielsweise ist die Verkehrsführung durch Schilder, aber auch durch die Fahrspurmarkierung gewährleistet, was bei Verschmutzung, Pfützen oder Schnee doppelte Sicherheit ergibt.

\section{WIRTSCHAFTLICHE INTERESSEN BEI DER AUTOMATION VON BETRIEBSABLÄUFEN}

Der autonome Busbetriebshof bietet ein wirtschaftliches Potenzial: Für die rund 150 Busse, die in Stuttgart-Gaisburg täglich gewartet werden, ergibt sich ein Kostenpotenzial von mehr als 100.000 Euro pro Jahr. Wichtiger als die Senkung der Personalkosten ist jedoch, dass die Mitarbeiter nicht durch Fahrzeiten und Fußwege blockiert werden, sondern für höherwertige Arbeiten zur Verfügung stehen, die die Qualität des Angebots für den Fahrgast erhöhen, aber derzeit aufgrund von Zeitmangel kurzgehalten werden (zum Beispiel ausführlichere Innenreinigung). Zudem werden durch automatisiertes Fahren Bagatellschäden durch Zusammenstöße reduziert.

Als weitere Anwendungen für autonome Nutzfahrzeuge sind Speditionsdepots, Verladeterminals oder Autobahnrastplätze denk- bar. Das Konzept aus Stuttgart-Gaisburg ist auch auf größere Betriebshöfe skalierbar. Besonders im Hinblick auf die Planung von neuen Betriebshöfen lassen sich die gewonnenen Erkenntnisse direkt einsetzen. So könnten in neuen Betriebshöfen Abholstationen am Tor vorgesehen werden, damit die Busse vom Busfahrer nicht mehr in der Abstellhalle entgegengenommen werden, sondern direkt an der Pforte. Dadurch werden Wege gespart und ein Betriebshof geschaffen, in dem kein menschliches Verhalten berücksichtigt werden muss.

\section{ZUSAMMENFASSUNG}

Das autonome Fahren bietet ein hohes Potenzial auf Betriebshöfen des öffentlichen Personennahverkehrs, da diese als eigenständige Einheit mit wiederkehrenden Arbeitsschritten gesehen werden können. Der Einsatz von autonom fahrenden Bussen kann den Personalaufwand enorm reduzieren. Die Sensoren und Aktoren zum Steuern eines Fahrzeugs besitzen bereits ausreichende Eigenschaften für die Manöver auf einem Betriebshof und bieten die Grundlage für das automatisierte Fahren. Durch den Einsatz von Standardtechnologie sowie den Betriebshofaufbau nach der StraBenverkehrsordnung reichen die Methoden und Fahrfunktionen von aktuellen Prototypen für den Einsatz auf dem Betriebshof aus.

\section{LITERATURHINWEISE}

[1] Straßenverkehrs-Zulassungs-Ordnung (StVZO) - $\$ 31$ Verantwortung für den Betrieb der Fahrzeuge. Moravia Druck + Verlag, Online: www.stvzo.de/verlag/ verlag.htm, Zugriff: 12.03.2018

[2] Daimler AG: The Mercedes-Benz Future Bus: The future of mobility. Daimler AG, Online: www.daimler.com/innovation/autonomes-fahren/future-bus.html, Zugriff: 12.03 .2018

[3] Lauber, A.; Glock, T.; Sax, E.; Wiedemann, M.: Analyzation and Evaluation of Vehicle and Infrastructure for Autonomous Driving on Public Transportation Depots. Tagung Commercial Vehicle Technology, Kaiserslautern, 2016 


\section{Repository KITopen}

Dies ist ein Postprint/begutachtetes Manuskript.

Empfohlene Zitierung:

Lauber, A.; Sax, E.; Wiedemann, M.

Autonomes Fahren auf dem Busbetriebshof.

2018. Automobiltechnische Zeitschrift, 120.

Doi: $10.5445 / I R / 1000083072$

Zitierung der Originalveröffentlichung:

Lauber, A.; Sax, E.; Wiedemann, M.

Autonomes Fahren auf dem Busbetriebshof.

2018. Automobiltechnische Zeitschrift, 120 (6), 74-77.

doi:10.1007/s35148-018-0047-y 